\title{
An in vivo comparative evaluation of graft uptake between chin and ramal graft in the reconstruction of atrophic anterior maxillary edentulous ridges for implant placement
}

\author{
S. Shanmuga Sundaram¹, Guru Prasad Thulasi Doss ${ }^{2}$, V.B. Krishna Kumar Raja ${ }^{3}$, Arun Ramaiah ${ }^{4 *}$ \\ ${ }^{1}$ Professor, ${ }^{2}$ Reader, ${ }^{3}$ Professor and Head, ${ }^{4}$ Senior Fellow, Dept. of Oral Maxillofacial Surgery, SRM Dental College, \\ Ramapuram, Chennai, Tamil Nadu, India
}

*Corresponding Author: Arun Ramaiah

Email: dr.arunramaiah@gmail.com

\begin{abstract}
Introduction: The goal of modern dentistry is to restore the patient to normal function, esthetics, comfort, speech and health, which can be brought by caries prevention or replacing of missing teeth. Loss of teeth may be devastating for the patient, both functionally and aesthetically. Complex anatomy, functional and aesthetic demands often make reconstruction very challenging as well as the multitude of complications that can arise during or after treatment.

Aim and Objectives: To evaluate the amount of bone resorption of mandibular autogenous block bone graft in reconstruction of atrophic anterior alveolar ridges.

Materials and Methods: 5 patients underwent harvesting of corticocancellous bone from Mandibular ramus and 5 patients underwent harvesting of corticocancellous bone from Mandibular chin region for Reconstruction of atrophic alveolar ridge. All patients were reviewed for follow-up for 5 months to evaluate the amount of resorption and acceptance of graft.

Results: In a follow up period of 5 months the mean graft resorption in ramal graft is $16.8 \%$ and mean graft resorption in symphysis graft is $18 \%$.

Conclusion: Minimally invasive approach, ease of application, volume of the graft and least postoperative morbidity are observed in ramal graft. The survival of the graft is better in ramal graft than the graft from the chin as the chin graft shows higher resorption rate. Patient compliance is favorable more towards the ramal graft than the chin graft as the immediate postoperative pain is high in symphysis region.
\end{abstract}

Keywords: Graft, Reconstruction, Implant, Ramus, Symphysis.

\section{Introduction}

Traditionally removable prosthesis or fixed partial dentures have been the treatment of choice in order to replace tooth loss permitting restoration of masticatory function, speech and aesthetics. Alveolar ridge resorption after tooth loss is a common phenomenon. After a tooth is extracted the alveolar ridge decreases in width and height very rapidly, with as much as $50 \%$ loss in width during the first year, two-thirds of which occurs in the initial 3 months. ${ }^{1}$ Often in clinical practice, the loss of a tooth does not coincide with replacement by a dental implant and there is frequently a lag of months to years before an edentulous site presents for therapy. Therefore, it is often required that we perform hard tissue ridge augmentation to increase bone volume prior to dental implant placement and restoration. Frequently, however, there is a lack of supporting bone in addition to the absent teeth due to disease, atrophy or trauma. Sufficient amount of underlying bone is required to stabilize the dental implant. $^{2}$ So that implants can be inserted in an ideal buccolingual and mesio-distal position with good axial inclination and to reshape the soft tissue contour. Different types of Bone grafts like Iliac crest, Ribs, Tibia, Fibula, Exostoses, Chin, Torus, Ramus, Tuberosity and grafting materials like Allografts, Alloplasts, Xenografts and are available for use in augmentation of Atrophic alveolar ridges. The autogenous bone grafts have been used for many years for ridge augmentation and are still considered the gold standard for jaw reconstruction. ${ }^{3}$ For most localized alveolar defects, as in reconstruction of atrophic alveolar ridges for implant placement, block bone grafts from the Symphysis and Ramus buccal shelf offer advantages over iliac crest grafts, including close proximity of donor and recipient sites, convenient surgical access, decreased donor site morbidity and decreased cost. ${ }^{4}$ The cortico-cancellous bone obtained from these sites facilitate faster vascular in growth, which results in rapid integration and less potential resorption during the healing period. ${ }^{5}$ Another important fact is that bone blocks harvested from intra membranous sites revascularize faster than those obtained from endochondral sites. ${ }^{6}$ Bone grafting in the atrophic edentulous alveolar ridge is mandatory for implant placement. Bone grafts have been obtained arbitrarily with the help of regular diagnostic protocols which end up in excess graft harvesting and donor site morbidity. Hence accurate graft resorption and required graft harvest can be assessed with advanced diagnostic aid such as Cone Beam CT and the amount of resorption determined, helps to obtain an appropriate sized graft in future for grafting cases. 


\section{Aims and Objectives}

To evaluate the amount of bone resorption of mandibular autogenous block bone graft in reconstruction of atrophic anterior alveolar ridges.

\section{Materials and Methods}

We included 11 patients in our study with anterior atrophic edentulous ridges. We evaluated the graft take up and amount of resorption in 5 patients ( 3 male and 2 female) with symphysis graft and 6 patients ( 3 male and 3 female) with ramal graft who had upper or lower anterior atrophic edentulous ridges. In symphysis group 3 male patients had upper anterior edentulous ridge and 2 female patients had lower anterior edentulous ridge. The reasons for bone loss being Periapical pathology in 1 patient, traumatic extraction in 2 patients and prolonged edentulism in 2 patients. In ramal group 3 male and 3 female had upper anterior edentulous ridge. The reason for bone loss being periapical pathology in 2 patients, traumatic pathology in 2 patients and prolonged edentulism in 2 patients. Out of which one patient was excluded as he was a chronic smoker. The subjects of this study were patients who visited for replacement of missing front teeth, 5 patients underwent harvesting of corticocancellous bone from Mandibular ramus and 5 patients underwent harvesting of corticocancellous bone from Mandibular chin region for Reconstruction of atrophic alveolar ridge. All patients were reviewed for follow-up, for 5 months to participate in the study to evaluate the amount of resorption and acceptance of graft. The patients were evaluated by the following methods: Clinical examination, Radiographs - OPG, IOPA, Cone beam CT, Patient interview. Healthy patients between the age group of 18 to 60 years, Patients with anterior edentulism, anterior edentulous patients with vertical or horizontal bone loss due to trauma, prolonged edentulism, cystic lesions etc. were included in the study. Smokers, aged patients, patients with poor oral hygiene, patients with un controlled diabetes, immunocompromised patients and patients with bleeding disorders were excluded.

\section{Results}

The harvested grafts were secured with a screw to ensure graft immobilization. In our study we analyzed the bone quality and amount of resorption with the help of Cone beam CT scan. In Symphysis group, patient 1 graft of $4 \mathrm{~mm}$ was placed, patient 2 graft of $4.2 \mathrm{~mm}$ placed, patient 3 graft of $5 \mathrm{~mm}$ thickness was placed, patient 4 graft of $4.5 \mathrm{~mm}$ was placed and in patient 5 graft of $5 \mathrm{~mm}$ was obtained. (Figure \& Table 1) In Ramal group patient 1 graft of $4.7 \mathrm{~mm}$ was placed, patient 2 graft of $3.1 \mathrm{~mm}$ was placed, patient 3 graft of $1.6 \mathrm{~mm}$ was placed, patient 4 graft of $5.2 \mathrm{~mm}$ was placed and patient 5 graft of $1.5 \mathrm{~mm}$ was placed. Preoperative width, immediate post-operative width, $4^{\text {th }}$ month post-operative width, resorption and resorption rate were evaluated in both the groups. (Figure \& Table 2)

Table 1: Data of patients with ramal graft

\begin{tabular}{|l|c|c|c|c|c|}
\hline Patient & $\begin{array}{c}\text { Pre-operative } \\
\text { width }\end{array}$ & $\begin{array}{c}\text { Post-operative } \\
\text { width }\end{array}$ & $\begin{array}{c}4^{\text {th }} \text { month post- } \\
\text { operative width }\end{array}$ & Resorption & Resorption\% \\
\hline 1 & $2.8 \mathrm{~mm}$ & $7.5 \mathrm{~mm}$ & $6.7 \mathrm{~mm}$ & $0.8 \mathrm{~mm}$ & $12 \%$ \\
\hline 2 & $3.6 \mathrm{~mm}$ & $6.7 \mathrm{~mm}$ & $5.5 \mathrm{~mm}$ & $1.2 \mathrm{~mm}$ & $21.8 \%$ \\
\hline 3 & $2.5 \mathrm{~mm}$ & $4.1 \mathrm{~mm}$ & $3.5 \mathrm{~mm}$ & $0.6 \mathrm{~mm}$ & $17.1 \%$ \\
\hline 4 & $2.0 \mathrm{~mm}$ & $7.2 \mathrm{~mm}$ & $6.3 \mathrm{~mm}$ & $0.9 \mathrm{~mm}$ & $14.2 \%$ \\
\hline 5 & $3.5 \mathrm{~mm}$ & $4.2 \mathrm{~mm}$ & $4.2 \mathrm{~mm}$ & $0.8 \mathrm{~mm}$ & $19 \%$ \\
\hline
\end{tabular}

Table 2: Data of patients with symphysis graft

\begin{tabular}{|l|c|c|c|c|c|}
\hline Patient & $\begin{array}{c}\text { Pre-operative } \\
\text { width }\end{array}$ & $\begin{array}{c}\text { Post-operative } \\
\text { width }\end{array}$ & $\begin{array}{c}4^{\text {th }} \text { month post- } \\
\text { operative width }\end{array}$ & Resorption & Resorption\% \\
\hline 1 & $2.9 \mathrm{~mm}$ & $6.9 \mathrm{~mm}$ & $6.0 \mathrm{~mm}$ & $0.9 \mathrm{~mm}$ & $15 \%$ \\
\hline 2 & $3.3 \mathrm{~mm}$ & $7.1 \mathrm{~mm}$ & $5.9 \mathrm{~mm}$ & $1.0 \mathrm{~mm}$ & $16 \%$ \\
\hline 3 & $2.5 \mathrm{~mm}$ & $6.5 \mathrm{~mm}$ & $5.3 \mathrm{~mm}$ & $1.2 \mathrm{~mm}$ & $22 \%$ \\
\hline 4 & $3.0 \mathrm{~mm}$ & $7.5 \mathrm{~mm}$ & $6.0 \mathrm{~mm}$ & $1.5 \mathrm{~mm}$ & $25 \%$ \\
\hline 5 & $2.3 \mathrm{~mm}$ & $7.3 \mathrm{~mm}$ & $6.8 \mathrm{~mm}$ & $0.8 \mathrm{~mm}$ & $11 \%$ \\
\hline
\end{tabular}




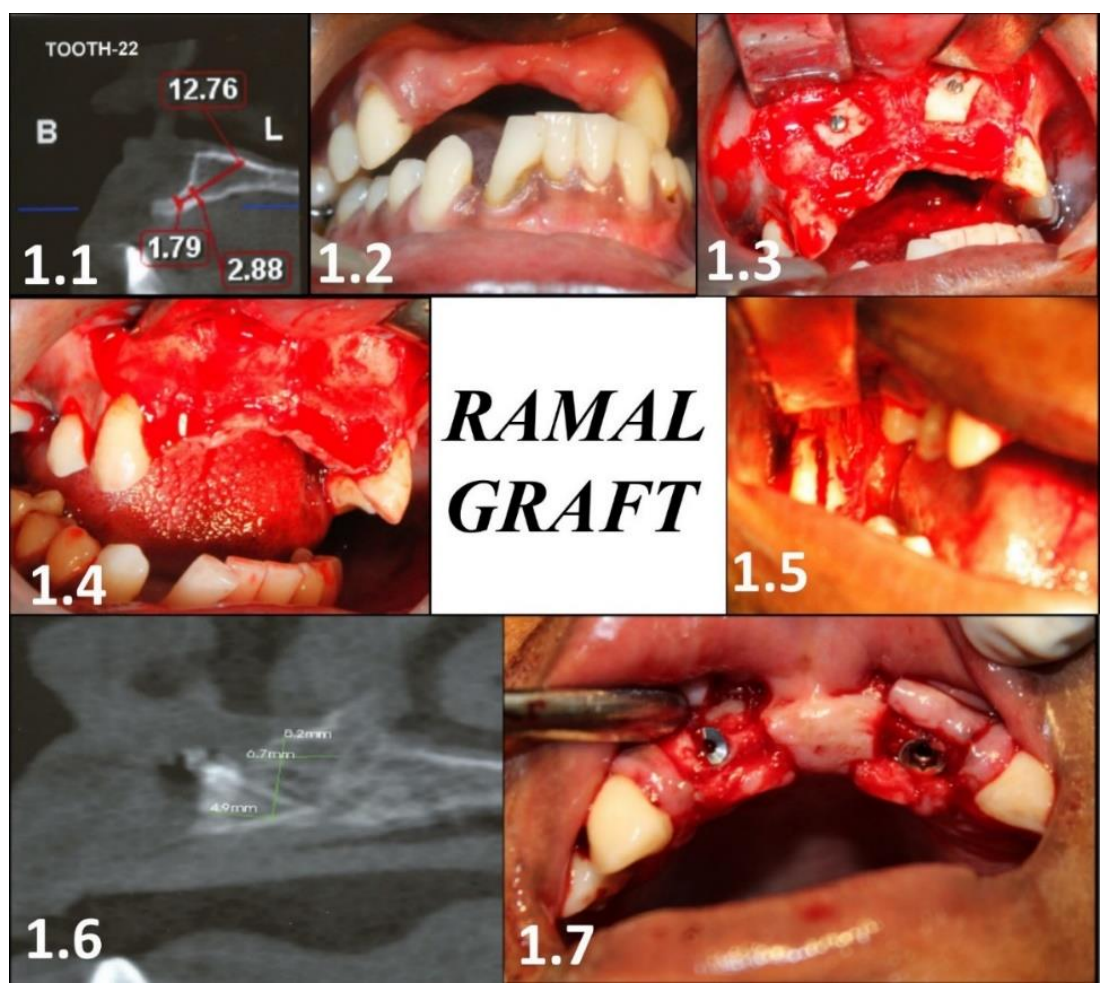

Fig. 1.1: Preoperative CBCT; Fig. 1.2: Preoperative intraoral photograph; Fig. 1.4: Elevation of the flap; Fig 1.5: Removal of the ramal graft; Fig. 1.3: Placement of graft, Fig 1.6: Postoperative CBCT; Fig. 1.7: Implant placement

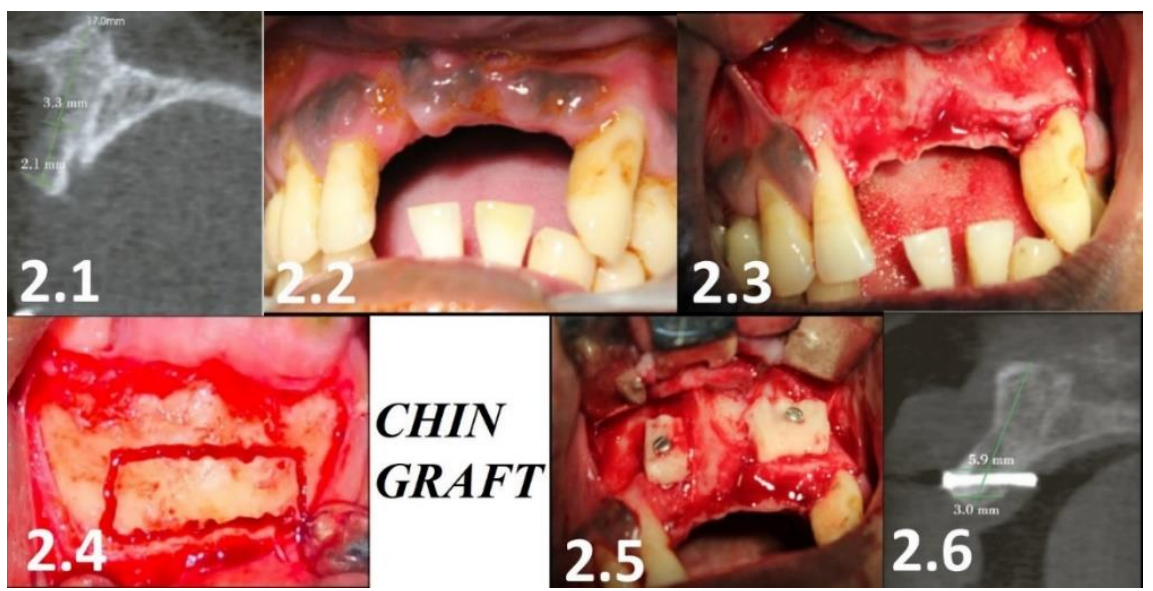

Fig. 2.1: Preoperative CBCT; Fig. 2.2: Preoperative intraoral photograph; Fig. 2.3: Elevation of the flap; Fig. 2.4: Removal of the chin graft; Fig. 2.5: Placement of graft; Fig. 2.6: Postoperative CBCT

\section{Discussion}

In our study the required volume is comfortable for implant placement which were obtained from symphysis or ramus region. Apart from the dimensional requirement, proximity of the donor site, devoid of second surgical site and similar osteogenic property of the graft also enables the surgeon in decision making to select these donor sites. ${ }^{7,8}$ Thus, autogenous bone grafts became popular and they have been used for many years for ridge augmentation. ${ }^{9}$ The use of autogenous bone grafts with Osseo integrated implants originally was discussed by Branemark and colleagues, who often used the distant sites like iliac crest, calvarium, rib and tibia. ${ }^{10}$ Our study was carried out to determine the amount of resorption of mandibular symphysis \& ramal block bone graft in anterior atrophic edentulous ridge for the future implant placement. This includes the amount of bone resorption in the due course, so that the exact amount of bone required for the augmentation alone can be harvested from the donor site in future which helps to minimize the donor site morbidity. Mean incidence of resorption of ramal graft is $16.8 \%$ whereas the resorption of symphysis graft is $18 \%$. Though there is a marginal distinction among the 
quantum of resorption, no qualitative significance was noticed in the bone while the placement of implants. Alveolar ridge resorption after tooth loss is a common phenomenon, alveolar ridge decreases in width and height very rapidly, nearly $50 \%$ loss in width within the first year in which $2 / 3 \mathrm{rd}$ of resorption occurs in the first 3 months. ${ }^{11-13}$ Most of the patients do not prefer dental implant treatment as a first choice due to various causes, primarily due to economic reasons. ${ }^{14}$ So, the patients often present to the clinician after a long edentulous span. In the study 9 patients were using removable dentures till the ridge augmentation procedure. The mean average edentulous period being 2 $1 / 2$ years ( 2 to 4$)$. As all the patients were having compromised dental rehabilitation both esthetically as well as functionally, the surgical augmentation followed by permanent implant prosthesis were planned. The age ranges for the patient group falls between 18 and 45 years, sex predilected to females and partly edentulous, the option for permanent restoration with implants gained more scope. Alveolar ridge augmentation is an inevitable procedure in many cases, where the qualitative and quantitative bone loss. ${ }^{15}$ Successful implant placement can be achieved in atrophic anterior regions by using block autografts. ${ }^{16}$ Versatility of the harvesting procedure, graft resorption and donor site morbidity are the main clinical concerns associated with autogenous grafting procedures. ${ }^{17}$ Based on such criteria, this prospective study has been carried out to compare the most commonly used donor sites like chin and ramus for harvesting the bone. Membranous grafts have shown less resorption than endochondral bone grafts, which suggests that intra oral donor sites may provide an advantage in harvesting block grafts for augmentation of the alveolar ridge, and they can be easily assessed in an office setting. ${ }^{18-20}$ With the aids of advanced imaging techniques such as Cone beam CT are also an effective diagnostic tool in the assessment of bone defects, bone resorption and greatly helpful in treatment. ${ }^{21}$ As the block bone graft augmentation in atrophic ridges for implant placement remains an attractive and simpler option, the procedure is widely applied by the clinicians rather than preferring the alloplasts. ${ }^{22-24}$ In symphysis donor site drawbacks are morbidity, which includes intra operative complications such as bleeding, mental nerve injury, soft tissue injury to the cheeks, lips, and tongue, block graft fracture, infection, and potential bicortical harvest, dysesthesia of the anterior mandibular dentition. ${ }^{25-27}$ Computed axial tomography can be used to determine osseous architecture without distortion. However, CT images have 3 major drawbacks, 1. High radiation dosage, 2. High degree of scattered radiation around metallic restorations and implants, 3. There is significant burnout of medullary bone which is directly proportional to the radiation dose. ${ }^{28}$

\section{Conclusion}

Considering the postoperative complications, though the symphysis is easier to approach, the invasion is greater as the muscles of the labial chin are transected and resutured. On the other hand, medial ramus can be approached transmucosally without any viable tissue injury. As the site is approached intraorally, the resultant defect doesn't cause any obvious facial disharmony. 1. Minimally invasive approach, ease of application, volume of the graft and least postoperative morbidity are observed in ramal graft. 2. The survival of the graft is better in ramal graft than the graft from the chin as the chin graft shows higher resorption rate. 3. Patient compliance is favorable more towards the ramal graft than the chin graft as the immediate postoperative pain is high in chin region.

\section{Limitations}

Periapical or panoramic $\mathrm{x}$ - rays have been used to evaluate the implant sites, there are limitations of these radiographs like distortion, magnification and missing 3rd dimension bone volume.

\section{Conflict of Interest: None}

\section{Source of Funding: None}

\section{References}

1. Antonio D'addonia, Hessam Nowzari. Intramembranous Autogenous Osseous Transplants in Aesthetic Treatment of Alveolar Atrophy. Journal of Periodontology. 2001;27:149-161.

2. Bernhard Pommertepper G, Gahleitner A, Zechner W, Watzekg. New Safety Margins for Chin Bone Harvesting Based on the Course of the Mandibular Incisive Canal in Ct, Clinical Oral Implants Research. 2008;19:1312-1316.

3. Brener Et. The Mandibular Ramus Donor Site. Australian Dental Journal. 2006;51(2):187-190.

4. Bradley. S. Mcallister, Kamran Haghighat. Bone Augmentation Techniques. Journal of Periodontology. 2007;78(3):377-396.

5. Cameron Y. S. Lee, Henry Hasegawa. Immediate Load And Esthetic Zone Considerations to Replace Maxillary Incisor Teeth Using A New Zirconia Implant Abutment in The Bone Grafted Anterior Maxilla. Journal of Oral Implantology. 2005;1:256-267.

6. Cintia mussi milani contar, João Rodrigo Sarot, Jayme Bordini, Jr, Gustavo Holtz Galvão, Gastão Vale Nicolau, Maria Angela Naval Machado. Maxillary Ridge Augmentation with Fresh-Frozen Bone Allografts, American Association of Oral and Maxillofacial Surgeons. J Oral Maxillofacsurg. 2009;67:1280-1285.

7. Federico Hernández-Alfaro, Eva Salvan-Garcia, Javier Mareque-Bueno, Eduard Ferres-Padró. "Envelope" Approach for Onlay Bone Grafting, Preliminary Surgical and Prosthetic Results. Med Oral Patol Oral Cir Bucal. 2004;16:45-49.

8. Gerry.M. Raghebar, Leo Meijndert, Wounter W.I. Kalk, Arjan Vissink. Maxillary Bone Grafting for Insertion of Endosseous Implants, Results After 12 - 124 Months. Clinical Oral Implant Research. 2001;12:279-286. 
9. Daniel Buserdula K, Hess D, Hirt Hp, Belser Uc. Localized Ridge Augmentation with Autografts and Barrier Membranes. Journal of Periodontology. 2000;19:151-163.

10. Daulton Keith, Maurice A. Salama. Ridge Preservation and Augmentation Using Regenerative Materials to Enhance Implant Predictability and Esthetics, Compendium. 2007;28(11):614-623.

11. David. C. Hatcher, Dial C, Mayorga C. Cone Beam Ct For Pre-Surgical Assessment of Implant Sites, Cda Journal. 2003;31(11):825-833.

12. Devorah Schwartz, Liran Levin. Intraoral Autogenous Block Onlay Bone Grafting for Extensive Reconstruction of Atrophic Maxillary Alveolar Ridges. Journal of Periodontology. 2005;76:636-641.

13. Domos.N. Mardas, Nikolaos Mardas, Vivek Chadha. Clinical Outcomes of Implants Following Lateral Bone Augmentation, Systematic Assessment of Available Options (Barrier Membranes, Bone Grafts, Split Osteotomy). Journal of Clinical Periodontology. 2008;35:173-202.

14. Gregory Taylor. Intra-Oral Autogenous Bone Grafting for Dental Implant Site Preparation, The Hong Kong Medical Diary. 2010;15:12-14.

15. Hadi antoun, Sitbon Jm, Martinez H, Missika P. A Prospective Randomized Study Comparing Two Techniques of Bone Augmentation, Onlay graft Alone or Associated with Membrane, Clinics of Oral Implantology and Research. 2001;12:632-639.

16. Ilarazerbade Lange Gl, Joldersma M, Bronckers Al, Burger Eh. Fate of Monocortical Blocks Grafted in The Human Maxilla, A Histological and Histomorphometric Study, Clinical Oral Implants Research. 2003;14:750756.

17. Joseph.A. Loenetti, Richard Koup. Localized Maxillary Ridge Augmentation with A Block Autograft for Dental Implant Placement, Case Reports, Implant Dentistry. 2003;12(3):217-226.

18. Kyung-Choon Oh, Cha Jk, Kim Cs, Choi Sh, Chai Jk, Jung Uw. The Influence of Perforating the Autogenous Block Bone and The Recipient Bed in Dogs. Part I, A Radiographic Analysis, clinical oral implant research, 2011;22:1298-1301.
19. Lars andersson. Patient Self-Evaluation of Intra-Oral Bone Grafting Treatment to The Maxillary Frontal Region, Dental Traumatology. 2008; 24:164-169.

20. Luca Cordaro, Ferdinandoboghi, Vincenzo Mirisola Di, Torresanto, Ferrucciotorsello. Reconstruction of The Moderately Atrophic Edentulous Maxilla with Mandibular Bone Grafts. Clin. Oral Impl Res. 2012:1-8.

21. Luca Cordaro, David Sarziamade, Massimo Cordaro. Clinical Results of Alveolar Ridge Augmentation with Mandibular Block Bone Grafts in Partially Edentulous Patients Prior To Implant Placement. Clin Oral Impl Res. 2002; 13: $103-111$

22. Luca Cordaro, David Sarziamade, Massimo Cordaro. Harvesting Bone in The Recipient Sites for Ridge Augmentation. Int J Periodontics Restorative Dent. 2008;28:411-419.

23. Mansipabari, Srinath N, Veeresh M. The Art of Block Grafting in The Preparation of Site for An Implant. Journal of Dental \& Allied Sciences. 2012;1:37-40.

24. Mario Rocuzzo, Guglielmoramieri, Marco Bunino, Sid Berrone. Vertical Alveolar Ridge Augmentation by Means of a Titanium Mesh and Autogenous Bone Grafts. Clinical Oral Implants Research. 2004;15:73-81.

25. Matteochiapasco, Paolo Casentini, Marco Zaniboni, Elena Corsi. Evaluation of Peri-Implant Bone ResorpTion Around Straumann Bone Level Implants Placed in Areas Reconstructed with Autogenous Vertical Onlay Bone Grafts. Clin. Oral Impl Res. 2011;23:1012-1021.

26. Michael A. Pikos. Mandibular Block Autografts For Alveolar Ridge Augmentation. Atlas Oral Maxillofacial Surgclin N Am. 2005;13:91-107.

27. Craig M. Mischdula K, Hess D, Hirt Hp, Belser Uc. Comparison of Intraoral Donor Sites for Onlay Grafting Prior To Implant Placement. Int J Oral Maxillofac Implants. 1997;12:767-776.

28. Robert.J. Millerwarren C. Edwards, Carlosboudet, Jonathan H. Cohen. Revised Maxillofacial Anatomy: The Mandibular Symphysis 3d. The International Journal of Dental Implants and Biomaterials. 2006;5:1-7. 